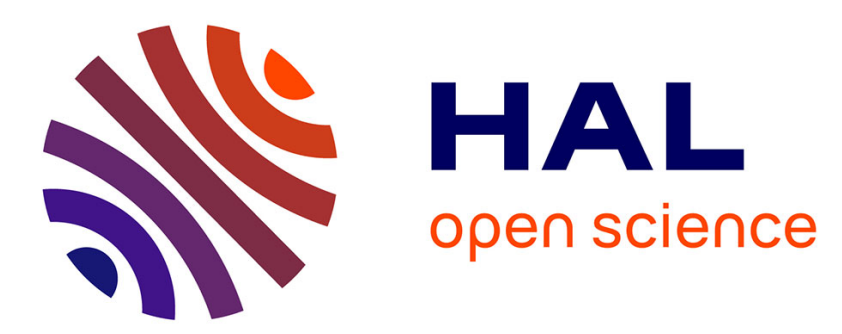

\title{
A new torsion pendulum technique to measure the twist elastic constant of liquid crystals
}

S. Faetti, M. Gatti, V. Palleschi

\section{To cite this version:}

S. Faetti, M. Gatti, V. Palleschi. A new torsion pendulum technique to measure the twist elastic constant of liquid crystals. Journal de Physique Lettres, 1985, 46 (18), pp.881-886. 10.1051/jphyslet:019850046018088100 . jpa-00232913

\section{HAL Id: jpa-00232913 https://hal.science/jpa-00232913}

Submitted on 1 Jan 1985

HAL is a multi-disciplinary open access archive for the deposit and dissemination of scientific research documents, whether they are published or not. The documents may come from teaching and research institutions in France or abroad, or from public or private research centers.
L'archive ouverte pluridisciplinaire HAL, est destinée au dépôt et à la diffusion de documents scientifiques de niveau recherche, publiés ou non, émanant des établissements d'enseignement et de recherche français ou étrangers, des laboratoires publics ou privés. 
Classification

Physics Abstracts

$61.30-62.20 \mathrm{D}-68.10 \mathrm{C}$

\title{
A new torsion pendulum technique \\ to measure the twist elastic constant of liquid crystals (*)
}

\author{
S. Faetti (**), M. Gatti and V. Palleschi \\ Dipartimento di Fisica dell'Universita' di Pisa, Piazza Torricelli 2, 56100 Pisa, Italy
}

(Reçu le 11 avril 1985, accepté sous forme définitive le 23 juillet 1985)

\begin{abstract}
Résumé. - Une nouvelle technique de mesure de la constante élastique de torsion des cristaux liquides nématiques est proposée. Cette technique est basée sur une mesure directe du couple exercé par le directeur sur les surfaces quand le nématique est soumis à un champ magnétique. Cette technique est appliquée à la mesure de la constante élastique $K_{22}$ du cristal liquide 4-n-pentyl-4'-cyanobiphényl (5CB).
\end{abstract}

\begin{abstract}
A new experimental method to measure the twist elastic constant of nematic liquid crystals is proposed. The method is based on a direct measurement of the torque which the nematic liquid crystal exerts on the surfaces when a magnetic field is applied. The reliability of this technique is tested by measuring the twist elastic constant of the nematic liquid crystal 4-n-pentyl-4'-cyanobiphenyl (5CB). The experimental results are compared with previous measurements performed by other authors.
\end{abstract}

\section{Introduction.}

Frank elastic constants [1] of nematic liquid crystals (LC) play an important rôle on the macroscopic properties of these materials. As a consequence of this a lot of experimental results on the elastic constants is now available in the literature. The elastic constants are usually obtained by measuring the threshold value of the magnetic field for the Freedericks transition [1] in thin nematic layers. This threshold value is given by

$$
H_{\mathrm{c} i}=\frac{\pi}{d} \sqrt{\frac{K_{i i}}{\chi_{\alpha}}}
$$

where $K_{i i}(i=1,2,3)$ is an elastic constant (splay, twist, bend), $\chi_{\alpha}$ is the anisotropy of the diamagnetic susceptibility and $d$ is the thickness of the layer. Rapini and Popoular [2] showed that a finite anchoring energy or a small misalignment of the director at the interfaces can considerably reduce the measured threshold field. This fact could explain some large discrepancies between experimental measurements of elastic constants [3-7] performed by different authors.

(*) Research supported in part by Ministero della Pubblica Istruzione and in part by Consiglio Nazionale delle Ricerche (Italy).

(**) Also Gruppo Nazionale di Struttura della Materia del CNR, Piazza Torricelli 2, 56100 Pisa, Italy. 
In a recent paper J. Grupp [8] proposed a new method for a direct determination of the twist elastic constant $K_{22}$. In his experiment a thin nematic layer is sandwiched between two parallel plane plates treated in such a way to induce a uniform orientation of the director along a single axis parallel to the plane of the plates. The upper plate is suspended by a thin quartz wire (torsion pendulum) and the lower plate is rotated to generate a twist of the director field. By this the upper plate is subjected to a mechanical torque and thus, it is rotated. By measuring the rotation angle Grupp obtained the twist elastic constant. This method requires a very long time to perform a measurement of one value of $K_{22}$ (one day).

In this paper we propose a new experimental method which is still based on a measurement of surface torques. In our case the distortion of the director-field and thus, the surface torque, is generated by a magnetic field. The time which is needed for measurements is very short (a few minutes for a single measurement of one elastic constant). The reliability of this technique is tested by measuring the twist elastic constant of 4-n-pentyl-4'-cyanobiphenyl (5CB). The experimental results are compared with those obtained by other authors.

\section{Principle of the method.}

Figure 1 shows schematically the experimental apparatus used to measure the twist elastic constant. The nematic LC (NLC in Fig. 1a) lies on the bottom of a cylindrical glass cell which is held at a fixed temperature by a water thermal bath $(\mathrm{T})$ within $10 \mathrm{mK}$. A circular glass plate (P) having a diameter of $15 \mathrm{~mm}$ and a thickness of $0.7 \mathrm{~mm}$ is dipped in the nematic sample. The plate is suspended by a thin $(30 \mu \mathrm{m})$ quartz wire $(\mathrm{W})$ fixed to a thin vertical glass tube $(\mathrm{Q})$, which is sealed to the upper surface of the plate. The suspension of the torsion pendulum can be rotated in the horizontal plane by means of a rotation stage $(R)$. The temperature is measured by means of a thermoresistor (TR in Fig. 1a). A uniform magnetic field ranging from 50 to $8800 \mathrm{G}$ can be applied along the horizontal $X$-axis. The rotation of the glass plate around the vertical axis is detected by measuring the deflection of a laser beam which is reflected by the mirror $M$. The

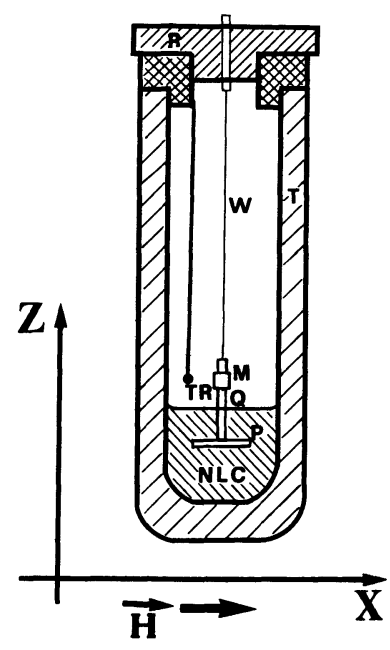

(a)

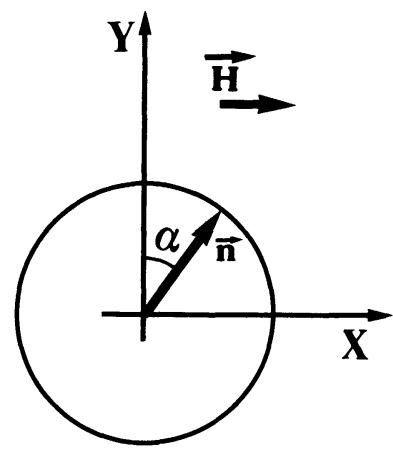

(b)

Fig. 1. - (a) Schematic vertical cross section of the experimental apparatus. NLC = nematic sample, $\mathbf{P}=$ circular glass plate, $\mathbf{Q}=$ vertical thin glass tube, $\mathbf{M}=$ mirror to detect small rotations of the torsion pendulum, $\mathbf{W}=$ thin quartz wire $(30 \mu \mathrm{m}), \mathbf{R}=$ rotation stage, $T=$ Thermostatic bath of circulating water, $\overrightarrow{\mathbf{H}}=$ magnetic field. (b) Schematic top view of the circular glass plate. $\overrightarrow{\mathbf{n}}$ indicates the director. 
accuracy of the measurement of the angular rotation of the torsion pendulum is about $10^{-4} \mathrm{rad}$. Both plane surfaces of the circular glass plate are treated by oblique evaporation of $\mathrm{SiO}$ [9] in such a way to obtain a uniform orientation of the director along a well defined axis in the $X-Y$ plane (easy axis) (see Fig. 1b). The magnetic field tends to orient the director parallel to the $X$-axis whilst the surface interactions orient the director along the easy axis at the surfaces of the glass plate. Therefore a twist of the director field occurs close to the surfaces within a thin layer having a thickness of the order of the magnetic coherence length $\xi \equiv 1 / H \sqrt{K_{22} / \chi_{\alpha}}$ (see Fig. 2). The excess of free energy due to the twist distortion can be relaxed by a suitable rotation of the torsion pendulum and thus, a torque is exerted on the glass plate.

This torque can easily be calculated by using the elastic theory of liquid crystals. After simple calculations we obtain $[1,8]$ :

$$
\tau=\sqrt{K_{22} \chi_{\alpha}} S H \cos \alpha
$$

where $H$ is the magnetic field and $\alpha$ is the angle between the director orientation on the surfaces and the $Y$-axis orthogonal to the magnetic field (see Fig. 1b), $S$ represents the treated part of the total surface of the circular glass plate. Equation (2) neglects completely the effects due to the not treated lateral circular surfaces of the plate $\mathbf{P}$ (boundary effects). The maximum spurious torque due to the lateral surface would occur if the director orientation on this surface was everywhere perpendicular to the magnetic field ( $Y$-axis). In this unreasonable case we estimate a spurious contribution to the torque of the order of the ratio of the untreated surface to the treated one $(\Delta S / S \sim 10 \%)$. However, the absence of a well defined easy direction on the lateral surface of the plate makes this latter condition very improbable and thus, we can estimate that the effective relative error is much lower than $5 \%$. However this error could be reduced by using a thinner glass plate.

As shown in equation (2) the surface torque is proportional to the cosine of the angle $\alpha$ and therefore depends for $\alpha \ll 1 \mathrm{rad}$ only weakly on the director orientation at the surface. In fact the relative error on $\tau$ due to the uncertainty of the angle $\alpha$ is $\frac{\Delta \tau}{\tau}=\Delta \alpha \operatorname{tg} \alpha$. For example, for $\alpha=0.349 \mathrm{rad}\left(\cong 20^{\circ}\right)$ and $\Delta \alpha=0.017 \mathrm{rad}\left(\cong 1^{\circ}\right)$ one obtains $\frac{\Delta \tau}{\tau}=0.6 \%$. Let us remark

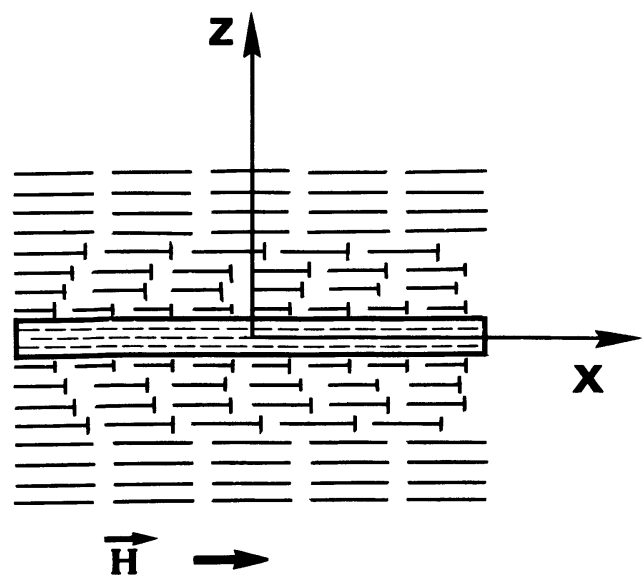

Fig. 2. - Schematic vertical cross section of the circular glass plate and of the director arrangement in the nematic sample. The system tends to relax the twist of the director-field by a proper rotation around the vertical $Z$-axis. 
that in the case of the Freedericks transition Rapini and Popoular [2] showed that an error of $1^{\circ}$ in the director orientation gives errors up to $10 \%$ in the threshold field. In the presence of a finite anchoring energy, the surface director angle $\alpha$ becomes a function of the magnetic field. However, as shown previously, the torque is weakly dependent on $\alpha$ when $\alpha \ll 1 \mathrm{rad}$. On the other hand if $\alpha \sim 1$ rad the torque $\tau$ becomes more sensible to small variations of the surface director angle and thus, it allows to perform a direct measurement of the anchoring energy. Finally let us notice that the present experiment furnishes $K_{22} \chi_{\alpha}$ (Eq. (2)) while the Freederiks transition technique furnishes a measurement of $K_{22} / \chi_{\alpha}$ (Eq. (1)). Current experimental values of $\chi_{\alpha}$ show often large discrepancies (of typically $8 \%$ ). Therefore a comparison between results for $K_{22}$ obtained by means of these two different experimental methods can also be helpful to discuss the accuracy of the $\chi_{\alpha}$ values.

\section{Experimental results.}

The nematic material used to test the reliability of our experimental apparatus is 4-n-pentyl4'-cyanobiphenyl (5CB) produced by British Drug House (BDH). This nematic LC shows a high purity and chemical stability [10]. As a matter of fact the clearing temperature of our sample remained stable within $0.1^{\circ} \mathrm{C}$ over several months. The uniformity of the alignment of the director at the surface was tested by the conoscopic technique.

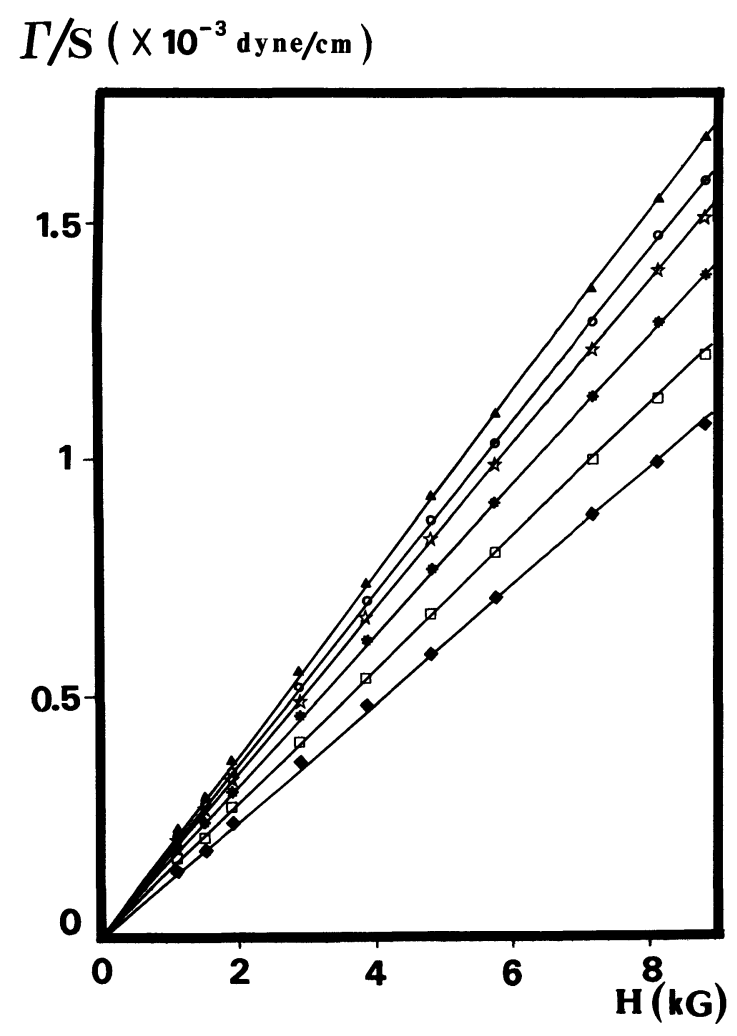

Fig. 3. - Value of the torque per unit surface versus the magnetic field. The parameter is the difference between the temperature $T$ and the clearing temperature $T_{\mathrm{c}}$ of the nematic LC. The angle between the director at the surface and the magnetic field is $72^{\circ}$. ( $\left.\diamond\right) T_{\mathrm{c}}-T=1.29{ }^{\circ} \mathrm{C}$, ( $\left.\square\right) T_{\mathrm{c}}-T=2.34^{\circ} \mathrm{C}$, (*) $T_{\mathrm{c}}-T=4.33^{\circ} \mathrm{C}$, (它) $T_{\mathrm{c}}-T=6.15^{\circ} \mathrm{C}$, (O) $T_{\mathrm{c}}-T=7.89^{\circ} \mathrm{C},(\mathbf{\Delta}) T_{\mathrm{c}}-T=9.80^{\circ} \mathrm{C}$. 
The amount of the torque $\tau$ is related to the angular rotation $\Delta \theta$ of the torsion pendulum by the simple relation

$$
\tau=k \Delta \theta
$$

where $k$ represents the torsion coefficient of the quartz wire. In the present experiment the torsion coefficient is $k=0.2073 \pm 0.0014$ dyne $\mathrm{cm} / \mathrm{rad}$.

In order to determine $K_{22}$ correctly it must be assumed that the twist of the nematic LC has everywhere the same sign. This is obtained by cooling the sample from the isotropic to the nematic phase in the presence of a high magnetic field $(5 \mathrm{kG})$.

Figure 3 shows the surface torque per unit surface versus the magnetic field for different temperatures. The linearity of the experimental results ensures a strong anchoring of the director at the surfaces in this range of temperatures. The quantity $K_{22} \chi_{\alpha}$ is obtained from the best fit of the experimental data according to equation (2). The uncertainty of $K_{22} \chi_{\alpha}$ is due to the following reasons : 1) the uncertainty of the surface $S(\Delta S / S<1 \%)$, 2) the uncertainty of the torsion coefficient $(\Delta K / K=0.7 \%)$, 3) the uncertainty of the measurement of the torque $(\Delta \tau / \tau<1 \%), 4)$ the error due to the boundary effects on the lateral surfaces of the glass plate $(\Delta \tau / \tau<5 \%), 5)$ the uncertainty of the magnetic field $H(\Delta H / H<1 \%)$. Therefore we estimate a $15 \%$ maximum relative error on the value of $K_{22} \chi_{\alpha}$. From $K_{22} \chi_{\alpha}$, one can obtain the elastic constant $K_{22}$ if $\chi_{\alpha}$ is known. Two different values of $\chi_{\alpha}$ for 5CB are available in the literature

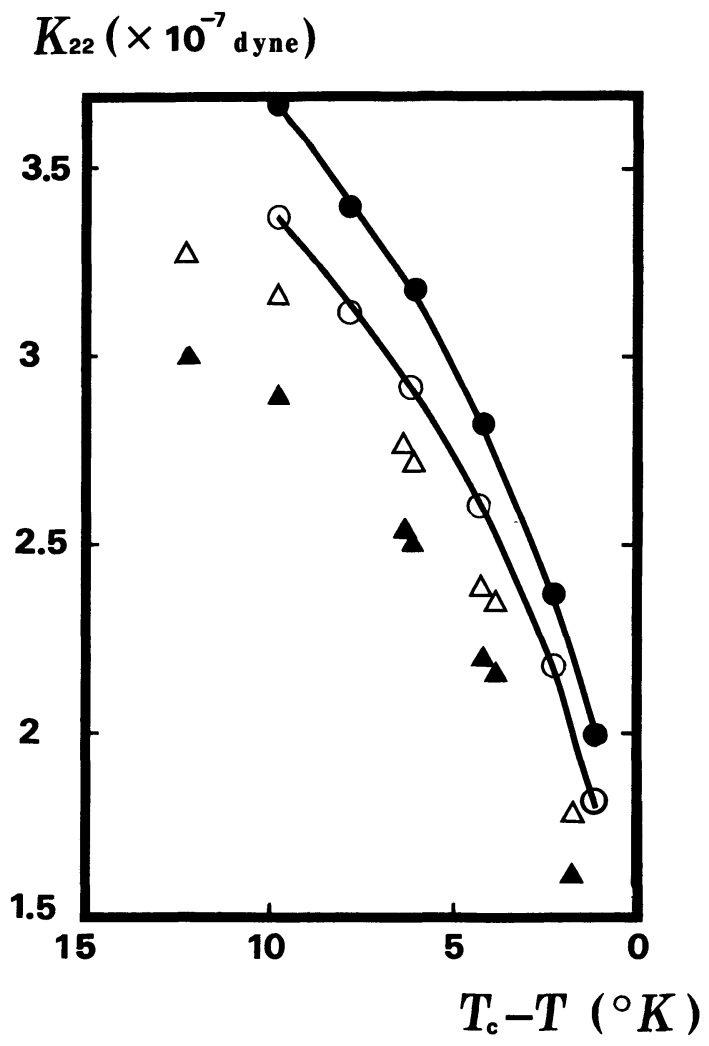

Fig. 4. - Values of the twist elastic constant of 5CB versus the temperature. Full and open dots correspond to our results obtained with $\chi_{\alpha}$ given in references [12] and [11], respectively. Full and open triangles correspond of the Madhusudana and Pratibha [3] results for $\chi_{\alpha}$ given in references [12] and [11], respectively. 
$[11,12]$ but which do not coincide. In particular the results of reference [11] are up to $8 \%$ higher than those of reference [12].

Figure 4 shows the temperature dependence of $K_{22}$. The full and open dots correspond to the values of $K_{22}$ obtained in the present experiment by using the $\chi_{\alpha}$ values of references [12] and [11], respectively. Measurements of $K_{22}$ for 5CB have - to our knowledge - only been performed by Madhusudana and Pratibha [3] who used the Freedericks technique (Eq. (1)) and evaluated $\chi_{\alpha}$ by the Pascal rule. Full and open triangles in figure 4 correspond to the values of $K_{22}$ which are here rescaled by the use of $\chi_{\alpha}$ given in references [12] and [11], respectively. Madhusudana and Pratibha estimate an accuracy of $15 \%$ of their experimental results. Our results obtained by using $\chi_{\alpha}$ given in reference [12] are $27 \%$ higher than those obtained by Madhusudana and Pratibha, whereas with $\chi_{\alpha}$ from reference [11] only a difference of $7 \%$ is found. Schad and Osman [4] have repeated the measurements of reference [3] for another member of the homologous series of cyanobiphenyls (7CB). They find a $K_{22}$ elastic constant which is also considerably higher $(20 \%)$ than that determined in reference [3]. Notice that higher values of the elastic constants are usually considered to be more precise since most of the error sources in a Freedericks experiment tend to reduce the measured threshold value. The large discrepancy between our experimental results and those by Madhusudana and Pratibha could be due to a systematic error of the experiment of reference [3] and to the uncertainty of the input parameter $\chi_{\alpha}$. New measurements of $K_{22}$ and $\chi_{\alpha}$ are necessary in order to get a more conclusive comparison with our experimental results.

\section{Conclusions.}

In this paper we propose a simple experimental method to measure the twist elastic constant of nematic LC. The method is based on a direct measurement of surface torques generated by a magnetic field. The main advantages of this method are : i) the experimental results are poorly sensitive to small misalignments of the director with respect to the magnetic field, ii) the presence of a finite anchoring energy of the director at the interfaces can easily be detected and, thus, it does not affect the accuracy of the measurement. The reliability of the method is shown by performing a measurement of $K_{22}$ of 5CB. The estimated accuracy of this measurement is of $15 \%$ and it could be increased to $5 \%$ by using a thinner glass plate.

\section{Acknowledgments.}

We thank Mr. Carlo Guidi and Mr. Manrico Taddei for their helpful technical collaboration.

\section{References}

[1] See, for example, De Gennes, P. G., The Physics of Liquid Crystals (Clarendon Press, Oxford) 1974.

[2] Rapini, A. and Popoular, M., J. Physique Colloq. 30 (1969) C4-54.

[3] Madhusudana, N. V. and Pratibha, R., Mol. Cryst. Liq. Cryst. 89 (1982) 249.

[4] Schad, Hp. and Osman, M. A., J. Chem. Phys. 75 (1981) 880.

[5] DE Jeu, W. H., Classen, W. A. P. and Spruijt, A. M. J., Mol. Cryst. Liq. Cryst. 37 (1976) 277.

[6] Haller, I., J. Chem. Phys. 57 (1972) 1400.

[7] Leenhouts, F., Von Der Wounde, F. and Dekker, A. J., Phys. Lett. A 58 (1976) 242.

[8] Grupp, J., Phys. Lett. A 99 (1983) 373.

[9] Janning, J. L., Appl. Phys. Lett. 21 (1972) 173.

[10] Gray, G. W., J. Physique Colloq. 36 (1975) C1-337.

[11] SCherrel, P. L. and Crellin, D. A., J. Physique Colloq. 40 (1979) C3-211.

[12] BuKA, A. and DE JEU, W. H., J. Physique 43 (1982) 361. 